\title{
Two Czech patients with familial adenomatous polyposis presenting mosaicism in $A P C$ gene
}

\author{
M. URBANOVA ${ }^{1, *}$, K. HIRSCHFELDOVA ${ }^{1,2}$, L. OBEIDOVA ${ }^{1}$, B. JANOSIKOVA ${ }^{2}$, J. LASTUVKOVA ${ }^{3}$, M. LUKAS ${ }^{4}$, J. KOTLAS ${ }^{1,2}$, J. STEKROVA ${ }^{1,2}$ \\ ${ }^{1}$ Institute of Biology and Medical Genetics, 1st Medical Faculty, Charles University, Prague, Czech Republic; ${ }^{2}$ General University Hospital, \\ Prague, Czech Republic; ${ }^{3}$ Department of Medical Genetics, Masaryk Hospital, Usti nad Labem, Czech Republic; ${ }^{4}$ Clinical Center Iscare, Prague, \\ Czech Republic
}

${ }^{*}$ Correspondence: marketa.urbanova@lf1.cuni.cz

Received July 31, 2018 / Accepted September 14, 2018

\begin{abstract}
During standard molecular diagnostic procedure, two Czech families with APC (Adenomatous polyposis coli gene) mosaicism have been detected. A woman with attenuated familial adenomatous polyposis (AFAP, OMIM \#175100) was recently inspected by next generation sequencing. Standard bioinformatics pipeline, restricted to variants with at least $20 \%$ of reads (for germline variants) would miss mutation p.G1412X (NM_000038.5) present in 17\% of reads. This novel variant was not present in any of her two children. Another woman with a clinical manifestation of attenuated FAP was tested 16 years ago without conclusive APC mutation found when denaturing gradient gel electrophoresis (DGGE), protein truncation test (PTT), multiplex ligation probe amplification (MLPA) and direct Sanger sequencing were applied. Recent inspection of her son showed clear mutation p.Q1062X (NM_000038.5, NP_000029.2) leading to premature stop codon. This finding led to re-evaluation of this protein position in his mother and detection of mosaicism (11\% of allele, $22 \%$ of heterozygous cells in blood), which was primarily overlooked. Mutations in both patients were confirmed by allele-specific real time PCR (AS qPCR). In both index patients it was possible to detect and quantify the mosaic allele in biological samples of polyps, adjacent colonic mucosa and buccal swabs. In cases of sporadic appearance of FAP, besides blood we plan to preferably inspect also other samples, where mosaic fraction might be under detection limit of bioinformatics pipelines $(<3 \%)$. For our future routine NGS sequencing analysis we will apply our in-house somatic variant detection pipeline to minimize the false negative calls when genes with high level of de-novo mutations are analyzed.
\end{abstract}

Key words: mosaic mutation, APC, FAP, AFAP, Czech patient

Familial adenomatous polyposis (FAP, OMIM \#175100) is a hereditary cancer disease caused mainly by heterozygous mutations in APC (gene ID 324). In classical FAP, patients start to have 100 s to 1000 s of polyps in colon during their second decade of life that clinically manifest during their third decade of life (gastrointestinal bleeding, abdominal pain, diarrhea). Although variably present, this syndrome has extracolonic manifestations including the development of benign and malignant tumor polyps of the gastric fundus and duodenum, osteomas, dental anomalies, congenital hypertrophy of the retinal pigment epithelium (CHRPE), soft tissue tumors, desmoid tumors, and associated cancers. About $80 \%$ of patients have a family history of FAP, whereas about $20 \%$ are carriers of de-novo mutation [1]. The life time risk of developing cancer is $100 \%$, if polyps are not resected early. APC abberation has a complete penetrance of colonic polyposis but variable penetrance of the extraco- lonic manifestations (desmoid tumors, lipomas, osteomas, medulloblastomas, gliomas, etc), also depending on the exact position of the APC mutation. The non-classical, attenuated FAP (AFAP), is usually presented with lower number of polyps in colon (below 100) and later age of onset (>40 years). When hereditary basis is very likely, but no $A P C$ mutation is detected, one may inspect other causative genes. There are other hereditary colon cancer syndromes whose clinical manifestation may overlap with FAP or AFAP and such diversity leads to diagnostic difficulties. MUTYH associated polyposis is an autosomal recessive disease caused by biallelic germline mutation of MUTYH glycosylase, a protein that functions in base-excision repair pathway. Another syndrome that may mislead the molecular diagnosis is an autosomal dominant polymerase-proofreading-associated-polyposis (PPAP) caused by heterozygous mutation in $P O L E$ and $P O L D 1$, two polymerases that function in nucleo- 
tide excision repair pathway. The most prevalent hereditary form of colon tumors caused by mutations in genes coding for mismatch repair proteins is Lynch syndrome. Patients with Lynch syndrome type I manifest colon cancers from the third decade of life, their adenomas (often proximal) tend to be larger with high grade of dysplasia that soon progresses into carcinoma. Extracolonic manifestations are more frequent than in FAP/AFAP, but are still rare. Overlapping genotype-phenotype correlations in hereditary colon cancer syndromes favor NGS technology in nowadays molecular diagnostic laboratories.

$A P C$ mutation detection rate strongly depends on the clinical manifestation of the index patient and his or her family history. In classic FAP, the rate is about $90 \%$. In AFAP with mild disease course, the rate is much lower $20-30 \%$ and thus more challenging. Approximately $10-25 \%$ of all identified germline APC mutations carriers have a de novo mutation [2]. Among those there is a substantial, but still underestimated proportion of mosaic carriers [3]. With the advantage of NGS technology which provides deep sequencing of selected regions, mosaicism in $A P C$ gene with relatively high rate of de novo mutations is detected more frequently [4-6]. Here, we present two Czech families with AFAP and APC mosaicism.

\section{Patients and methods}

Clinical data. Under standard laboratory procedure, index patient blood or DNA are sent to laboratory under specified indicative criteria. These include: family history of colon cancer, young age at diagnosis, detection of several polyps, adenomas and carcinoma in colon and rectal tissue, or extracolonic cancers associated with FAP/AFAP disease.

First case (index patient of family \#1, Figure 1) is a young woman $\left({ }^{*} 1975\right)$ without family history of FAP but with clinical symptoms of AFAP disease. She was diagnosed at the age 41 when she suffered from extensive anemia and colonoscopy revealed polyposis $(<100$, from caecum till sigmoideum, semicircular tumorous lesion in the right colon was found, adenocarcinoma was proved histologically). She underwent subtotal colectomy (7/2016); she had no extracolonic manifestations. Her daughter $\left({ }^{\star} 2006\right)$ and her son $\left({ }^{\star} 2012\right)$ are still too young to manifest any signs of disease. DNA was obtained from all three members of this family. The index patient also kindly provided her buccal swab and biopsies from rectal polyps.

Second case (index patient of family \#2, Figure 1) is a woman $\left({ }^{*} 1959\right)$ with possible FAP/AFAP that underwent hemicolectomy due to polyposis and stage I cancer in left colon. Her son (Figure 1, fam. \#2, III/2, ${ }^{\star} 1981$ ) presented clinically as FAP sy. at the age 20 with abundance $(>500)$ of very small polyps $(2-3 \mathrm{~mm})$ in the left colon and starting endothelial prominences in the right colon. In 2017, after he underwent total colectomy, our laboratory received his DNA for APC gene analysis. At the age of 25 he underwent total colectomy with ileal pouch. Index patient's daughter (Figure 1 , fam. \#2, III/1, ${ }^{\star} 1979$ ) is healthy, no polyps were detected during periodical colonoscopic inspections. Thanks to cooperation with the family, we also obtained buccal swab, colon polyp and adjacent colon mucosa from the index patient.

Molecular genetics. All members of family \#1 and \#2 (Figure 1) gave permission (informed consent approved by ethical committee) to inspect their biological samples for $A P C$ mutation detection. Peripheral blood is the basic source of DNA for genetic analysis. Routine molecular diagnostic of $A P C$ changes over time according to recent guidelines.

Molecular genetic testing of carriers was previously based on prescreening of DNA isolated from blood for germline mutation. Firstly, denaturing gradient gel electrophoresis (DGGE) of amplicons covering all 15 exons and adjacent intron boundaries was applied. Secondly, highly sensitive protein truncation test (PTT) was applied, and thirdly, all positive captures were confirmed by Sanger sequencing method.

At present, we start our analysis with MLPA (P_043 MRC Holland) to inspect large gene rearrangements. The expected number of $A P C$ chromosomal rearrangements that can be detected is around $6 \%$ of all APC mutations in most populations $[7,8]$. We confirm our positive MLPA finding of exon deletion/duplication by another method. Firstly, we apply direct sequencing to prove that there is no sequence variant under the MLPA hybridizing probe that could cause artificial exon drop-out. Secondly, we perform real time PCR to apply other independent method for quantity measures of exon dosage. In case of negative finding, we perform NGS panel sequencing that harbors the main genes concerned in heritable forms of colon cancer syndromes. All NGS variants within selected panel of genes are then confirmed by direct Sanger sequencing.

In family \#1, DNA of the index patient was tested for the presence of APC mutation by the NGS panel FAP MASTR v1.0 (Multiplicom) that harbors APC and MUTYH (gene

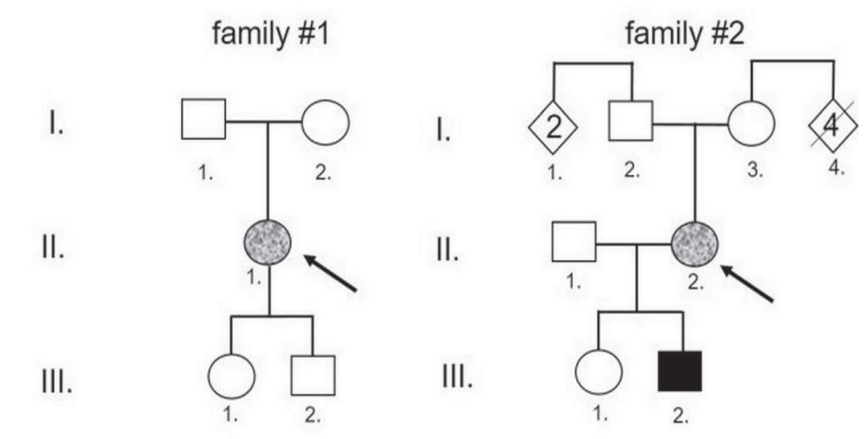

Figure 1. Pedigree of family \#1 and \#2. Pedigrees show the position of index patients in their family. Grey color represents a mosaic carrier, whereas black color represents clear heterozygous carrier of APC mutation (fam.\#2, III/2). Arrow indicates index patient. 
ID 4595). Amplicon based NGS library was sequenced on MiSeq platform (Illumina) with $2 \times 250 \mathrm{bp}$ reads. Bioinformatic evaluations of NGS data were firstly done by software MiSeq Reporter (Illumina). Short after were those data reevaluated by an in-house constructed bioinformatic pipeline that allowed us to monitor variants with low frequencies (above 0.01). Demultiplexed reads from Illumina MiSeq sequencer were trimmed and quality-filtered; the adaptor sequences used during amplicon-based library preparation were clipped off using Cutadapt v1.14 [9] and reads with low quality were afterwards filtered by Trimmomatic v0.36 [10]. Preprocessed reads were mapped against the hg38 reference genome using BWA-MEM with default settings (BWA v0.7.12, from bwa.kit software package) [11]. BAM files were sorted and indexed with SAMtools v1.6 [12]. Variant calling was performed using FreeBayes v1.1.0-9-g09d4ecf [13] with min-alternatefraction option lowered to 0.01 . Annotation and functional effect prediction of variant were done by SnpEff v4.3r [14] and SnpSift v4.3r [15].

In family \#2, DNA of the index patient underwent $A P C$ mutation screening in year 2002 with no conclusive mutation capture (DGGE negative, PTT negative, MLPA negative (P_43 B1 MRC Holland), Sanger sequencing negative). Her DNA was stored in the DNA bank. In 2017 her son's DNA was sent to laboratory for APC mutation detection.

Once the mutation of both families was identified, we applied allele-specific real-time PCR to perform confirmation based on qualitative and quantitative measures. The quantification was based on comparison of $\mathrm{Ct}$ values between target (wild type/mutated APC allele) and reference (amplicon of approximately same size, similar efficiency). Allele specific primers were designed by online available tools (primer3, WASP [16]) and slightly modified to optimize PCR reaction. For each of the mutation three pairs of primers were designed and tested. The highest specificity for mutation of family \# 1 was reached with primers: forward 5'-CCCACTCATGTTTAGCAGAT3' with reverse (APCwt specific) 5'-ATAATGCCACTTACCATTCC-3' and reverse (APCmut specific) 5'-ATAATGCCACTTACCA-TTCA-3' for p.G1412X, c. 4234 G>T (NM_000038.5). For family \#2 mutation, the following primers were selected: forward 5'-ATGAGCAGTTGAACTCTGGA-3' with reverse (APCwt specific) 5'-CTTGATTGTCTTTGCTCACTCTG-3' and reverse (APCmut specific) 5'-CTTGATTGTCTTTGCTCACTCTA-3' for p.Q1062X, c.3184 C>T (NM_000038.5). For AS qPCR experiment we used Hot FirePol Eva Green master mix (no ROX) (Solis Biodyne, Estonia). Experiment was run on LightCycler $\odot 480$ machine (Roche, USA). Result $\mathrm{Ct}(\mathrm{Cp})$ data were independently evaluated by LightCycler Instrument Software (Roche, USA) and Microsoft Excel (Microsoft, USA).

When the qPCR assay was established, we also obtained other tissues. DNA analysis of buccal swab, polyp and healthy tissue could help in determining different proportions of $A P C$ mutation in different germline layers. We hypothesized that the highest portion of $A P C$ mutation will be present in polyp tissue representing the endoderm layer of developing embryo.

\section{Results}

We have detected two mosaic deleterious APC mutations strongly associated with familial adenomatous polyposis syndrome in both families. Table 1 summarizes the results of our study.

In the family \#1: index patient is a carrier of somatic mosaic mutation p.G1412X, c. 4234 G>T (NM_000038.5), which was primary detected by the NGS method. Various biological samples represent different mosaic fraction. The highest amount of mutated cells was recorded in blood (34\%) and, surprisingly, the lowest in one of the rectal polyp samples $(9 \%)$ (Table 1).

In the family \#2, the index patient is a carrier of somatic mosaic mutation p.Q1062X, c.3184 C>T (NM_000038.5), [17]. Her mosaic variant was detected only thanks to the positive capture of APC mutation in her son. His DNA was screened by MLPA (P_43 D1 MRC Holland). Due to discrepancy in one of the MLPA probes, direct sequencing of the $15^{\text {th }}$ exon followed and clearly causative heterozygous mutation was identified. The carrier of the mosaic had the highest amount of mutated cells in the colon polyp.

Both families' mutations were confirmed consequently by AS qPCR. Primers specific for mutated allele showed the highest specificity, whereas APC wild-type-specific primers allowed undesirable amplification of mutated allele. Table 1 shows the summary of amount of mutated alleles/ cells in different samples and tissues, mainly quantified by AS qPCR. All examined samples (blood, buccal swab, polyps and adjacent mucosa) were positive for detectable mosaic fraction. Although, none of the samples is composed only of the cells of its origin (ectodermal for buccal cells, endodermal for colon tissue and mesodermal for the blood cells), the majority of them should. We have confirmed the clinical diagnosis in both of these patients.

\section{Discussion}

During our routine molecular genetic testing, we have identified APC mutation as a mosaic in two Czech index patients with attenuated FAP disease.

Different methods led to the identification of mosaicism. In the family \#1, it was the NGS method with aberrant allele detection in lower number of reads. Whereas in the family $\# 2$, it was the mutation capture in the DNA of index patient's son by MLPA method that led us to revision of the specific position of exon 15 of APC in the index patient herself.

Our previous standardized NGS data processing filtered out variants with aberrant allele fraction lower than $20 \%$ which represented satisfactory threshold for germline heterozygous variants. As the time went by, we tried to improve 
Table 1. Genetic information of the two Czech families with APC mosaicism.

\begin{tabular}{|c|c|c|c|c|}
\hline Patient/Sample & $\begin{array}{l}\text { Mutation } \\
\text { (NM_000038.5) }\end{array}$ & $\begin{array}{c}\text { Method of } \\
\text { quantification }\end{array}$ & $\begin{array}{c}\text { Quantity } \\
\text { (\% aberrant alleles/cells measured } \\
\text { by APC mutation allele count) }\end{array}$ & Clinical Appearance \\
\hline \multicolumn{5}{|l|}{ family \#1 } \\
\hline \multicolumn{5}{|l|}{ index patient (Figure 1, fam. \#1, II/1) } \\
\hline \multirow[t]{2}{*}{ blood } & \multirow[t]{2}{*}{ c.4234 G>T, p.G1412X } & NGS & $17 / 34$ & \multirow{8}{*}{$\begin{array}{l}\text { woman, age at diag- } \\
\text { nosis } 41 \text {, extensive } \\
\text { anemia, polyposis, } \\
\text { semicircular tumorous } \\
\text { lesion in the right } \\
\text { colon }\end{array}$} \\
\hline & & AS QPCR & $17 / 34^{*}$ & \\
\hline polyp1 & c.4234 G>T, p.G1412X & AS QPCR & $4.5 / 9.0$ & \\
\hline polyp2 & c. 4234 G>T, p.G1412X & AS QPCR & $11.9 / 23.8$ & \\
\hline polyp3 & c. 4234 G>T, p.G1412X & AS QPCR & $6.0 / 12.0$ & \\
\hline polyp4 & c. 4234 G>T, p.G1412X & AS QPCR & $6.3 / 12.6$ & \\
\hline buccal swab1 & c. 4234 G>T, p.G1412X & AS QPCR & $11.5 / 23.0$ & \\
\hline buccal swab2 & c. 4234 G>T, p.G1412X & AS QPCR & $12.3 / 24.6$ & \\
\hline $\begin{array}{l}\text { daughter (Figure 1, fam. \#1, III/1) } \\
\text { blood }\end{array}$ & none & AS QPCR & $0 / 0^{* *}$ & healthy \\
\hline $\begin{array}{l}\text { son (Figure 1, fam. \#1, III/2) } \\
\text { blood }\end{array}$ & none & AS QPCR & $0 / 0^{* *}$ & healthy \\
\hline family \#2 & & & & \\
\hline index patient (Figure 1 , fam. $\# 2$, II/ & & & & \\
\hline blood & c.3184 C>T, p.Q1062X & $\begin{array}{l}\text { MLPA/Sanger seq. } \\
\text { AS QPCR }\end{array}$ & $\begin{array}{l}\text { N.A. } \\
13.5 / 27\end{array}$ & $\begin{array}{l}\text { woman, age at diag- } \\
\text { nosis } 42 \text {, polyposis }\end{array}$ \\
\hline polyp & c.3184 C>T, p.Q1062X & AS QPCR & $32.5 / 65$ & $(<100)$, stage I colon \\
\hline buccal swab & c.3184 C>T, p.Q1062X & AS QPCR & $21 / 42$ & cancer in left colon \\
\hline healthy colon mucosa & c.3184 C>T, p.Q1062X & AS QPCR & $6,9 / 13,8$ & \\
\hline son (Figure 1, fam. \#2, III/2) & & & & man, FAP diagnosed at \\
\hline blood & c.3184 C>T, p.Q1062X & AS QPCR & $50 / 100$ & 20 , left colon polyposis \\
\hline daughter (Figure 1, fam. \#2, III/2) & & & & \\
\hline blood & none & AS QPCR & $0 / 0^{* * *}$ & healthy \\
\hline
\end{tabular}

Table represents quantity of aberrant alleles/cells in a specified tissue of two families with mosaic in APC gene. AS qPCR - allele specific real time polymerase chain reaction for mutated APC allele, ${ }^{*}$ - primary mutated allele measure based on NGS, ${ }^{* *}$ - exact number 0.003 considered as 0 , ${ }^{* *}$ - exact number 0.07 considered as 0 .

our NGS data processing to obtain even lower fractions of aberrant alleles since we started to work with somatic mutations present in tumors. New setup of bioinformatics pipeline and variant filtering allowed us to monitor aberrant alleles with frequency as low as 0.01 , although we see 0.03 as satisfactory. As we allow to record low frequency variants, we obtain many false positive calls. Those could be filtered out by comparisons with an in-house database to minimize library and platform-specific technical artefacts. Such bioinformatics processing allowed us to capture mosaic APC mutation in family \#1 with $17 \%$ of aberrant alleles.

MLPA method that identified a heterozygote mutation in an index patient's son (Figure 1, fam.\#2, III/2) appeared as lowering (0.66) one of the probes (namely APC-18 (WT)w). MLPA kit B1 and D1 slightly differ in the probe composition. D1 version is boosted by addition of extra probes aiming at identifying frequently aberrated positions of the large APC gene exon 15; among others the mutation at position c.3184. This probe was not included when the DNA sample of index patient (Figure 1, fam.\#2, II/2) was analyzed by MLPA, B1 version, and what is more, MLPA is not a suitable method to capture low amounts of mosaic allele. Sanger sequencing of the large exon 15 confirmed clearly causative mutation in heterozygote constitution in the son of index patient. Without this scenario, mosaic mutation would be missed.

The primary tissue for genetic testing is peripheral blood. This material is easily retrieved and for all hereditary forms of colon cancer diseases blood should provide sufficient quantity and quality for most of the applied diagnostic methods. If there is a somatic mutation present in the blood DNA with frequency close to $5 \%$, mainly NGS method is sensitive enough to capture it. Even lower mutation burden is possibly captured (less than 1\%), but the limit relies on the sequencing platform, read depth and bioinformatics tools that search for variants within sequenced region. All these parameters have an effect on frequency of false positive calls $[18,19]$.

Blood screening in both index patients showed 17\% (34\% of cells) and $13.5 \%$ (27\% of cells) of variant allele frequencies, respectively. However, in different patients, if only blood is inspected for the presence of mutation, mosaicism could be missed due to very low, or even null presentation 
of mutated allele. In studies $[4,5,20]$, mosaic variant allele fraction in blood of FAP/AFAP patients was frequently null or very close to $0 \%(12.5 \%, 0-9 \%, 0-4 \%$ - respectively). On the other hand, presence of a small fraction of mutated allele in blood points to the fact that mutation must had occurred before separation of endoderm (colon tissue) and mesoderm (blood) which in humans is set to occur before the third week after fertilization. Primordial germ cells that are forming future gametes have their origin in primary ectoderm [21]. Detection of mosaicism in blood thus makes it more probable for the future gametes to carry mutation. However, when a proband has less than $50 \%$ of somatic mosaicism, as in this case and others, the inheritance risk for any offspring depends on the level of mosaicism in the parental germ cell $[3,22]$. In our case, both of the index patients were women and presence of mutation in gametes was not possible to test. In index patient of family \#2, germ cells did carry mutation, since son is an affected carrier. For our future routine practice, we plan to ask the clinicians to provide not only blood, but also the buccal swab (or polyp when possible) in cases with FAP/AFAP symptoms but no family history of such disease.

In the index patient of family \#1, mosaic was detected in all examined biological materials. Surprisingly, colon polyps did not represent the tissue with the highest rate of mutated cells $(9-23.8 \% ; 14.35 \%$ on average). In this case, it was the primary blood DNA that carried the most affected cells (34\%). Each polyp had a different level of mutated cells $(4.5 \%$, $6 \%, 6.5 \%, 11.9 \%)$. It was even reported that polyp could be without a mosaic mutation [5] where only 10/16 resected adenomas carried such mutation. However, the situation in which the mutated cells are more frequent in adenomas than in the blood is more common $[4,6]$. Unfortunately, we could not obtain the sample of an unaffected colon mucosa for comparison. Index patient's children are not carriers of APC mutation. Although, the presence of this mutation in the mother's gametes is not excluded.

In the second family, mosaic was also detected in all examined biological materials of the index patient. Here, the highest proportion of mutated cells was observed in the polyp (65\%). Higher presence of mutated cells in buccal tissue $42 \%$ opposed to $13.8 \%$ in healthy colon mucosa is speculative and may reflect the heterogeneity of mosaicism in randomly selected material for examination. However, the level of $13.8 \%$ is sufficient to eventually develop polyp with dysplastic cells, as seen in the index patient \#1. The overall abundance of mosaic cells in index patient $\# 2$ reflects the sooner occurrence of the de-novo mutation in the developing embryo. The fact, that the son of this index patient is a heterozygote carrier, and that the daughter is not a carrier, confirms the presence of $A P C$ mutation in the primordial germ cells.

Adenoma samples of our index patients showed both higher and lower fraction of mosaic compared to blood. Therefore, we cannot conclude that adenoma samples are preferential for DNA diagnostics in sporadic cases of FAP/ AFAP patients. However, majority of mosaic reported cases shows higher fraction of mutated cells in adenomas compared to blood. In a work of Spier et al. [20], they showed results of a polyp that contained $92 \%$ of DNA having specific mutation previously not detected in blood. Such a high number $92 \%$ (exome NGS: $23 / 25$ reads) is not reached even in a heterozygous carrier. This adenoma sample presents a clone where, most probably, the other allele carrying APC has been lost. Interestingly, not all adenoma DNA samples from $A P C$ mosaic carrier contain the mosaic mutation [5]. Authors provide explanation through co-occurrence of sporadic adenomas within a mosaic environment. For that reason, at least two independent adenoma samples for screening purposes would minimize the risk of false negative result in sporadic FAP cases.

Index patient fam.\#1 is a carrier of p.G1412X, c. $4234 \mathrm{G}>\mathrm{T}$ and index patient fam.\#2 is a carrier of p.Q1062X, c.3184 C>T (NM_000038.5), both localized in the large exon 15 of the APC. Position p.1412 is well situated in the mutation cluster region (MCR; codons 1286-1513), whereas, position p.1062 is more upstream in the 15 -amino acid repeats central region, but both reside at the $\beta$-catenin binding region [23]. The majority of FAP patients have nonsense or frame-shift mutation in this exon leading to aberrant protein length (www.HGMD.org). The exact position of such mutation in the heterozygote constitution is somehow predictive for the clinical outcome [24-26]. Our mosaic cases present with an attenuated colorectal disease according to the age of onset (41 and 42) and/or polyp burden $(<100)$, despite the fact that the position of the mutation in the APC would have been expected to result in classical FAP disease. According to ClinVar database (https://www.ncbi.nlm.nih.gov/clinvar/), both of these mutations are strongly associated with pathologic effect on final APC protein.

Clinically, both of the index patients suffered from symptoms resembling FAP/AFAP disease at the age 41 and 42 , respectively. Higher age of onset and number of polyps categorizes these patients into a group of AFAP patients. According to literature, APC mosaic carriers are more AFAP. The age distribution is $30-61$ years in [20], and 26-50 years in [27]. In other diseases where mosaicism was confirmed, patients manifest rather less severe clinical symptoms. For example, mosaicism of FGFR3 (geneID 2261) causes common inborn defect (1:1000) epidermal nevi in human skin [28] opposed to achondroplasia, profound skeletal disorder caused by complete heterozygote FGFR3 mutation. However, literature also shows cases of mosaicism where symptoms were rather severe as a patient with Li-Fraumeni syndrome [29]. It seems that there is no conclusive general clinical picture of affected individuals based on the percentage of affected mosaic cells detected from blood. Mosaic carriers show broad spectrum of clinical signs from severe classic FAP to attenuated form of the disease. The level of mosaicism in leukocytes shows no consistent correlation with disease severity [20]. 
Somatic mosaicism in many other genes is also being described [30-32]. The huge progress in detection methods, namely sequencing provided by NGS technology, brings new cases of mosaicism in other diseases and shows much more frequent occurrence in human genome than previously thought [33]. In colorectal cancer disease, the occurrence of mosaicism is estimated to $20-25 \%$ of all de-novo mutation in sporadic FAP/AFAP cases [3]. Overlapping phenotypes between the colorectal syndromes have been recognized and this makes targeted molecular testing for single genes less favorable. Instead, there is a gaining interest for multi-gene panel-based approaches detecting not only SNVs and indels, but also CNVs in the same assay [34]. The NGS approach shows major benefits on costs and time required compared to conventional Sanger sequencing [35].

Detection of somatic mutation from NGS data could be a real challenge for routine laboratory practice. As mentioned above, the positive captures avoiding false positive calls rely strongly on the sequencing platform, read depth and bioinformatics tools that search for variants within sequenced region $[18,19]$. Our laboratory personnel developed an in-house Linux-based pipeline that opposed to previously applied available commercial software (MiSeq Reporter, set on default on germline variants), focuses on detection of variants of frequencies even lower than $10 \%$. Although, this pipeline allows capture of mutations with minimal occurrence, variants within the frequency range $1-3 \%$ seem to be sequencing artifacts. For this reason, we record variants that reach frequencies above $3 \%$.

With the advantage of NGS technology that provides deep sequencing of selected regions, the report of mosaicism in APC with relatively high rate of de novo mutations is detected more frequently $[4,5,20]$. It is very important for the offspring of mosaic carriers to be aware of the presence of deleterious mutation in APC gene since early therapeutical management may prevent cancer development.

We have identified two Czech families with mosaic mutation in APC gene by two different methods. First index patient was identified by NGS sequencing. Whereas, second index patient would be overlooked without the fact that this mutation was transmitted to her son as a clear heterozygous mutation. With the advantages of NGS technique, we expect to record mosaicism during our routine laboratory molecular diagnostics more frequently with appropriate bioinformatics evaluation. Since the mosaicism may be behind many sporadic cases of putative FAP disease, it is important to screen also other biological samples (buccal swabs or polyps where possible) where primary APC mutation screening is without any possibly damaging variant capture.

Acknowledgements: This study was supported by a Grant Progres Q28/LF1 and a GAČR P302/12/G157, Prague. The authors are grateful for the cooperation with the patients, their family members and their clinicians.

\section{References}

[1] BURT R, NEKLASON DW. Genetic testing for inherited colon cancer. Gastroenterology 2005; 128: 1696-1716. https:// doi.org/10.1053/j.gastro.2005.03.036

[2] ARETZ S, UHLHAAS S, CASPARI R, MANGOLD E, PAGENSTECHER $C$ et al. Frequency and parental origin of de novo APC mutations in familial adenomatous polyposis. Eur J Hum Genet 2004; 12: 52-58. https://doi.org/10.1038/ sj.ejhg. 5201088

[3] HES FJ, NIELSEN M, BIK EC, KONVALINKA D, WIJNEN JT et al. Somatic APC mosaicism: an underestimated cause of polyposis coli. Gut 2008; 57: 71-76. https://doi.org/10.1136/ gut.2006.117796

[4] IWAIZUMI M, TAO H, YAMAGUCHI K, YAMADA $\mathrm{H}$, SHINMURA K et al. A novel APC mosaicism in a patient with familial adenomatous polyposis. Hum Genome Var 2015; 2: 15057. https://doi.org/10.1038/hgv.2015.57

[5] JANSEN AM, CROBACH S, GEURTS-GIELE WR, VAN DEN AKKER BE, GARCIA MV et al. Distinct Patterns of Somatic Mosaicism in the APC Gene in Neoplasms From Patients With Unexplained Adenomatous Polyposis. Gastroenterology 2017; 152: 546-549 e543. https://doi.org/10.1053/j. gastro.2016.10.040

[6] SPIER I, KERICK M, DRICHEL D, HORPAOPAN S, ALTMULLER J et al. Exome sequencing identifies potential novel candidate genes in patients with unexplained colorectal adenomatous polyposis. Fam Cancer 2016; 15: 281-288. https://doi.org/10.1007/s10689-016-9870-z

[7] JARRY J, BRUNET JS, LAFRAMBOISE R, DROUIN R, LATREILLE J et al. A survey of APC mutations in Quebec. Fam Cancer 2011; 10: 659-665. https://doi.org/10.1007/s10689011-9468-4

[8] KERR SE, THOMAS CB, THIBODEAU SN, FERBER MJ, HALLING KC. APC germline mutations in individuals being evaluated for familial adenomatous polyposis: a review of the Mayo Clinic experience with 1591 consecutive tests. J Mol Diagn 2013; 15: 31-43. https://doi.org/10.1016/j. jmoldx.2012.07.005.

[9] MARTIN M. Cutadapt removes adapter sequences from high-throughput sequencing reads. EMBnet.journal 2011; 17: 10-12.

[10] BOLGER AM, LOHSE M, USADEL B. Trimmomatic: a flexible trimmer for Illumina sequence data. Bioinformatics 2014; 30: 2114-2120. https://doi.org/10.1093/bioinformatics/btu170

[11] LI H. Aligning sequence reads, clone sequences and assembly contigs with BWA-MEM. arXiv 2013. https://arxiv.org/ abs/1303.3997v2

[12] LI H, HANDSAKER B, WYSOKER A, FENNELL T, RUAN $J$ et al. The Sequence alignment/map (SAM) format and SAMtools. Bioinformatics 2009; 25: 2078-2079. https://doi. org/10.1093/bioinformatics/btp352

[13] GARRISON E, MARTH G. Haplotype-based variant detection from short-read sequencing. arXiv 2012. https://arxiv. org/abs/1207.3907v2 
[14] CINGOLANI P, PLATTS A, WANG LE L, COON M, NGUYEN $T$ et al. A program for annotating and predicting the effects of single nucleotide polymorphisms, SnpEff: SNPs in the genome of Drosophila melanogaster strain w(1118); iso-2; iso-3. Fly (Austin) 2012; 6: 80-92. https://doi. org/10.4161/fly.19695

[15] CINGOLANI P, PATEL VM, COON M, NGUYEN T, LAND SJ et al. Using Drosophila melanogaster as a Model for Genotoxic Chemical Mutational Studies with a New Program, SnpSift. Front Genet 2012; 3: 35. https://doi. org/10.3389/fgene. 2012.00035

[16] WANGKUMHANG P, CHAICHOOMPU K, NGAMPHIW C, RUANGRIT U, CHANPRASERT J et al. WASP: a Webbased Allele-Specific PCR assay designing tool for detecting SNPs and mutations. BMC Genomics 2007; 8: 275. https:// doi.org/10.1186/1471-2164-8-275

[17] LAGARDE A, ROULEAU E, FERRARI A, NOGUCHI T, QIU J et al. Germline APC mutation spectrum derived from 863 genomic variations identified through a 15 -year medical genetics service to French patients with FAP. J Med Genet 2010; 47: 721-722. https://doi.org/10.1136/jmg.2010.078964

[18] XU C, NEZAMI RANJBAR MR, WU Z, DICARLO J, WANG Y. Detecting very low allele fraction variants using targeted DNA sequencing and a novel molecular barcodeaware variant caller. BMC Genomics 2017; 18: 5. https://doi. org/10.1186/s12864-016-3425-4

[19] XUAN J, YU Y, QING T, GUO L, SHI L. Next-generation sequencing in the clinic: promises and challenges. Cancer Lett 2013; 340: 284-295. https://doi.org/10.1016/j.canlet.2012.11.025

[20] SPIER I, DRICHEL D, KERICK M, KIRFEL J, HORPAOPAN $S$ et al. Low-level APC mutational mosaicism is the underlying cause in a substantial fraction of unexplained colorectal adenomatous polyposis cases. J Med Genet 2016; 53: 172179. https://doi.org/10.1136/jmedgenet-2015-103468

[21] GILBERT SF. (Eds.). Developmental Biology, 6th Edition. Sinauer Associates, Sunderland 2000, p 695. ISBN 9780878932436.

[22] ZLOTOGORA J. Germ line mosaicism. Hum Genet 1998; 102: 381-386.

[23] LEOZ ML, CARBALLAL S, MOREIRA L, OCANA T, BALAGUER F. The genetic basis of familial adenomatous polyposis and its implications for clinical practice and risk management. Appl Clin Genet 2015; 8: 95-107. https://doi. org/10.2147/TACG.S51484

[24] NAGASE H, MIYOSHI Y, HORII A, AOKI T, OGAWA M et al. Correlation between the location of germ-line mutations in the APC gene and the number of colorectal polyps in familial adenomatous polyposis patients. Cancer Res 1992; 52: 4055-4057.
[25] WALLIS YL, MACDONALD F, HULTÉN M, MORTON JE, MCKEOWN CM et al. Genotype-phenotype correlation between position of constitutional APC gene mutation and CHRPE expression in familial adenomatous polyposis. Hum Genet 1994; 94: 543-548.

[26] KOHLER EM, DERUNGS A, DAUM G, BEHRENS J, SCHNEIKERT J. Functional definition of the mutation cluster region of adenomatous polyposis coli in colorectal tumours. Hum Mol Genet 2008; 17: 1978-1987. https://doi. org/10.1093/hmg/ddn095

[27] ARETZ S, STIENEN D, FRIEDRICHS N, STEMMLER S, UHLHAAS $S$ et al. Somatic APC mosalicism: a frequent cause of familial adenomatous polyposis (FAP). Hum Mutat 2007; 28: 985-992. https://doi.org/10.1002/humu.20549

[28] HAFNER C, VAN OERS JM, VOGT T, LANDTHALER M, STOEHR R et al. Mosaicism of activating FGFR3 mutations in human skin causes epidermal nevi. J Clin Invest 2006; 116: 2201-2207. https://doi.org/10.1172/JCI28163

[29] PROCHAZKOVA K, PAVLIKOVA K, MINARIK M, SUMERAUER D, KODET R et al. Somatic TP53 mutation mosaicism in a patient with Li-Fraumeni syndrome. Am J Med Genet A 2009; 149a: 206-211. https://doi.org/10.1002/ ajmg.a.32574

[30] REITEROVA J, STEKROVA J, MERTA M, KOTLAS J, ELISAKOVA $\mathrm{V}$ et al. Autosomal dominant polycystic kidney disease in a family with mosaicism and hypomorphic allele. BMC Nephrol 2013; 14: 59. https://doi.org/10.1186/14712369-14-59

[31] REKONDO J, ROBLEDO-INARRITU M, VADO Y, PEREZ DE NANCLARES G, ARSS F. [Marfan Syndrome Caused by Somatic Mosaicism in an FBN1 Splicing Mutation]. Rev Esp Cardiol 2016; 69: 520-521. https://doi.org/10.1016/j. rec.2016.01.018

[32] WEINSTEIN MM, KANG T, LACHMAN RS, BAMSHAD M, NICKERSON DA et al. Somatic mosaicism for a lethal TRPV4 mutation results in non-lethal metatropic dysplasia. Am J Med Genet A 2016; 170: 3298-3302. https://doi. org/10.1002/ajmg.a.37942

[33] VELTMAN JA, BRUNNER HG. De novo mutations in human genetic disease. Nat Rev Genet 2012; 13: 565-575. https://doi.org/10.1038/nrg3241

[34] ROHLIN A, RAMBECH E, KVIST A, TORNGREN T, EIENGARD F et al. Expanding the genotype-phenotype spectrum in hereditary colorectal cancer by gene panel testing. Fam Cancer 2017; 16: 195-203. https://doi.org/10.1007/ s10689-016-9934-0

[35] SIMBOLO M, MAFFICINI A, AGOSTINI M, PEDRAZZANI C, BEDIN C et al. Next-generation sequencing for genetic testing of familial colorectal cancer syndromes. Hered Cancer Clin Pract 2015; 13: 18. https://doi.org/10.1186/ s13053-015-0039-9 